\title{
Mobility properties of the Hermes transposable element in transgenic lines of Aedes aegypti
}

\author{
Ryan C. Smith • Peter W. Atkinson
}

Received: 10 August 2009/Accepted: 9 April 2010/Published online: 3 July 2010

(C) The Author(s) 2010. This article is published with open access at Springerlink.com

\begin{abstract}
The Hermes transposable element has been used to genetically transform a wide range of insect species, including the mosquito, Aedes aegypti, a vector of several important human pathogens. Hermes integrations into the mosquito germline are characterized by the noncanonical integration of the transposon and flanking plasmid and, once integrated, Hermes is stable in the presence of its transposase. In an effort to improve the post-integration mobility of Hermes in the germline of Ae. aegypti, a transgenic helper Mosl construct expressing Hermes transposase under the control of a testis-specific promoter was crossed to a separate transgenic strain containing a target Hermes transposon. In less than $1 \%$ of the approximately 1,500 progeny from jumpstarter lines analyzed, evidence of putative Hermes germline remobilizations were detected. These recovered transposition events occur through an aberrant mechanism and provide insight into the non-canonical cut-and-paste transposition of Hermes in the germ line of Ae. aegypti.
\end{abstract}

R. C. Smith · P. W. Atkinson

Graduate Program in Cell, Molecular, Developmental Biology, University of California, Riverside, CA 92521, USA

Present Address:

R. C. Smith

Department of Molecular Microbiology and Immunology, Malaria Research Institute, Johns Hopkins University Bloomberg School of Public Health, 615N. Wolfe Street, Room W4008, Baltimore, MD 21205, USA

\section{P. W. Atkinson $(\square)$}

Department of Entomology, Institute for Integrative Genome Biology, University of California, 900 University Avenue, Riverside, CA 92521, USA

e-mail: peter.atkinson@ucr.edu
Keywords Hermes . Transposable element . Germline remobilization $\cdot$ Beta-tubulin $\cdot$ Aedes aegypti

\section{Introduction}

Transposable elements (TEs) can successfully modify the genome of an organism through transposition. Five Class II transposable elements have been used to genetically transform a wide range of medically and agriculturally significant insect pests (Robinson et al. 2004). Transposons are genetic tools for functional genomic studies and have been proposed to be used in novel genetic control strategies to combat the transmission of mosquito-borne pathogens (James 2000). To date, five TEs have been used to genetically transform mosquitoes: Mos1, Minos, piggyBac, Tn5, and Hermes. Originally described within the house fly, Musca domestica (Atkinson et al. 1993), the Hermes element has since been used to transform the mosquitoes Aedes aegypti (Jasinskiene et al. 1998) and Culex quinquefasciatus (Allen et al. 2001). Germline integrations into both of these species are characterized by non-canonical cut-and-paste mechanisms in which plasmid sequence flanking the element is also integrated into the genome (Allen et al. 2001; Jasinskiene et al. 2000; Pinkerton et al. 2000). Previous studies have indicated that upon integration in the Ae. aegypti germline, Hermes is stable in the presence of transposase supplied by the Drosophila melanogaster hsp70 promoter (O'Brochta et al. 2003). Autonomous Hermes elements have, however, demonstrated the ability to readily transpose by a traditional cut-and-paste mechanism within the soma (O'Brochta et al. 2003).

This is in direct contrast to the behavior of Hermes in D. melanogaster, the stable fly, Stomoxys calcitrans, the Mediterranean fruitfly Ceratitis capitata and the butterfly 
Bicyclus anynana in which Hermes integrates through a standard cut-and-paste mechanism (Marcus et al. 2004; Michel et al. 2001; O'Brochta et al. 2000; O'Brochta et al. 1996). Furthermore, once integrated, Hermes elements can remobilize within the $D$. melanogaster germline at a rate of approximately 0.03 jumps per element per generation (Guimond et al. 2003). Beyond insects, Hermes has been developed as an efficient transposon genetic tool in Schizosaccharomyces pombe (Evertts et al. 2007).

Germline remobilization experiments with other insect Class II elements also display similar behavior within the mosquito germline as compared to D. melanogaster. The piggyBac element is a valuable tool for enhancer trapping in D. melanogaster (Horn et al. 2003) and in the red flour beetle Tribolium castaneum (Lorenzen et al. 2003; Lorenzen et al. 2007), but within Ae. aegypti piggyBac is completely immobilized in somatic and germline tissue subsequent to the initial integration (O'Brochta et al. 2003; Sethuraman et al. 2007). Similar experiments performed in Ae. aegypti for a transgenic Mosl element demonstrated that germline remobilization was extremely rare (1 in $\sim 14,000$ ) (Wilson et al. 2003). Within mosquitoes this apparent inability for transgenic transposons to be remobilized following transformation has been observed for the Minos element in Anopheles stephensi, in which germline remobilization events were not detected, although evidence of somatic mobility was recovered (Scali et al. 2007).

The application of TEs as genetic resources within mosquitoes for the use of enhancer trapping, gene trapping and mutagenesis would complement the availability of whole genome projects in three mosquito species and would have a significant impact on the study of disease transmission or vector/pathogen interactions and so serve as a valuable tool to study mosquito physiology and development. For example the identification of novel regulatory regions that direct expression within a specific tissue of interest, such as the midgut, fat body or salivary glands, could be harnessed to a desired effector gene to prevent pathogen transmission through the infected female mosquito. Furthermore, the creation of a heritable mutant through the insertion of an element in or near a specific gene could provide critical information regarding mosquito biology and development, and serve to highlight differences between mosquitoes and insects that do not require a blood meal for egg development.

Another proposed application of TEs has been to utilize the ability of an element to increase its copy number in a genome in order to drive a desired transgene into a population in a similar fashion to the spread of the $P$ element in natural D. melanogaster populations (Anxolabehere et al. 1988). Population replacement strategies for mosquitoes rely on the release of a sub-population refractory to disease transmission yet require a method to ensure the spread of the refractory allele within the natural population. Intrinsic to the implementation and feasibility of these strategies is the ability of a TE to efficiently remobilize within the germline.

Previous attempts to remobilize transposable elements within mosquito species have relied on the use of the D. melanogaster heat shock promoter to express transposase and we reasoned that the use of a germline, testis-specific promoter from Ae. aegypti may help to increase levels of transposase expressed within the germline. While a previous study used the Ae. aegypti nanos promoter and UTR sequences to direct the expression of Mos 1 transposase to regions of the developing fertilized egg in which germline tissue develop, the use of the nanos regulatory sequences to direct remobilization of incumbent Mos 1 elements was not examined (Adelman et al. 2007). Here we couple the $A a \beta 2 \mathrm{t}$ promoter to the Hermes transposase to regulate its expression within the male germline, and examine whether it can remobilize a previously integrated Hermes element. This Hermes element was found to remobilize, however several of the recovered transpositions appear to have remobilized by a non-canonical mechanism previously demonstrated with the Hermes element in Ae. aegypti (Pinkerton et al. 2000). While there is experimental support for some of these occurring in the germline, we believe that the most conservative explanation is that many may have occurred early in somatic development. Whether remobilization through aberrant transposition is confined to the germline genome and the mechanistic basis of this remains unknown.

\section{Materials and methods}

\section{Plasmid construction}

The Hermes ORF was amplified using TripleMaster ${ }^{\mathrm{TM}} \mathrm{Taq}$ (Eppendorf, Hamburg, Germany) from an existing pCRSHermes6 plasmid containing the wild type transposase from Musca domestica, using the primers AgeI Hermes start (5'-ATAACCGGTATGCAGAAAATGGACAATTTGGA AGTG- $\left.3^{\prime}\right)$ and NotI Hermes stop (5'-ATAGCGGCCGCTT ATATATCTAATTTACAAAAATTTTTGTAAAAGG-3'). The $\sim 1.8 \mathrm{~kb}$ PCR product was purified with the QIAquick PCR Purification Kit (Qiagen, Valencia, CA) according to manufacturer's protocol, and then digested with AgeI and NotI. Concurrently, a p $\beta 2$ DsRed2-1 (Smith et al. 2007) construct containing $959 \mathrm{bp}$ of the Ae. aegypti $\beta 2$ tubulin (Aaß2t) promoter was digested with AgeI and Not I, excising the DsRed ORF, and replaced by the Hermes ORF. The entire Aaß2t promoter-Hermes-SV40 poly A fragment was then amplified using TripleMaster ${ }^{\mathrm{TM}}$ Taq (Eppendorf, Hamburg, Germany) with the primer pair AscI $B 2$ proB (5'-ATAGGCGCGCCCCTTAGATTTTTTGTTTAAGTA TTTCGAAG- $\left.3^{\prime}\right)$ and $A s c$ I SV40 poly A (5'-TATGGCGC 
GCCGCCTTAAGATACATTGATGAG-3') to create AscI ends flanking each side of the fragment. The $\sim 3.0 \mathrm{~kb}$ fragment was purified using the QIAquick PCR Purification Kit (Qiagen, Valencia, CA), cloned into pGEM $^{\circledR}$-T easy (Promega, Madison, WI) and the sequence verified. Correctly sequenced clones of the Aaß2t promoter-Hermes-SV40 poly A fragment were excised with $A s c \mathrm{I}$ and inserted into the $A s c$ I site in pMos[3xP3-DsRed] to generate the M3DB2Her construct.

Before injection, DNA from the M3DB2Her plasmid was isolated with the Endofree Plasmid Maxi Kit ${ }^{\circledR}$ (Qiagen, Valencia, CA) according to the manufacturer's protocol.

\section{Embryo microinjection}

The microinjection of Ae. aegypti embryos was performed as previously described (Morris et al. 1989). The Hermes transposase expressing helper line was established by the co-injection of $500 \mathrm{ng} / \mu \mathrm{l}$ of the Mosl-based element, M3DB2Her, and $300 \mathrm{ng} / \mu \mathrm{l}$ of the pKhsp82-Mos1 (Coates et al. 1995) helper plasmid. Surviving $G_{o}$ animals were crossed as adults to wild type individuals after injection, and the resultant $G_{1}$ progeny were screened for the presence of DsRed using the appropriate filter sets. All images were captured using a Nikon DXM1200 digital camera mounted on a Leica MZ FLIII fluorescence stereomicroscope.

\section{Establishment of crosses}

Using the previously established non-autonomous Hermes reporter line pHer[A5C-EGFP] (Pinkerton et al. 2000), and the Hermes-expressing helper lines based from the M3DB2Her construct, transgenic individuals from both lines were crossed. Resulting reporter/helper progeny segregating with both fluorescent markers were collected based on the presence of both transgenic constructs. Reporter/helper hybrid individuals were then crossed to wild type individuals of the opposite sex or allowed to selfcross with other reporter/helper siblings. EGFP expressing progeny were collected from each cross and stored individually until later processing.

\section{RNA Isolation}

Mosquito samples were isolated from pupae, adults, testes, and dissected males from both transgenic Hermes-producing helper lines (M3DB2Her). Testes were dissected from adult mosquitoes, 1-3 days post eclosion, in chilled Aedes physiological saline (Hayes 1953) and collected along with the remaining dissected carcasses. All tissue samples were frozen in liquid nitrogen and stored at $-80^{\circ} \mathrm{C}$ until later use. Total RNA was isolated using TRIzol® (Invitrogen, Carlsbad, CA) according to the manufacturer's protocol, then treated with RNase-free DNase I for $1 \mathrm{~h}$ at $37^{\circ} \mathrm{C}$. Samples were further purified by phenol/chloroform extraction and quantified using the $\mathrm{DU}^{\circledR}-20$ spectrophotometer (Beckman Coulter, Fullerton, CA).

\section{Reverse-transcriptase PCR (RT-PCR)}

Two micrograms of total RNA from the M3DB2Her helper lines underwent reverse-transcription essentially as previously described (Smith et al. 2007). To examine the presence of Hermes transposase expression, a 500 base pair fragment of the Hermes ORF was amplified with the primers Hermes ORF 1283 F (5'-GACAGTTTGTTGCC CAATCCTGTTTCTC-3') and Hermes ORF 1782 R (5'-GG CACTACACAGCATTTTTTTCTATCCGC-3'). To standardize between reactions, cytoplasmic actin from Ae. aegypti was amplified as a control as previously described (Smith et al. 2007). RT-PCR was carried out using MasterTaq (Eppendorf, Hamburg, Germany) for a total of 28 cycles. Samples were run on agarose gels and visualized with the AlphaImager ${ }^{\mathrm{TM}}$ IS-2200 imaging system (Alpha Innotech, San Leandro, CA).

\section{Southern Blots}

To verify the digest pattern of the parental pHer[A5CEGFP] parental construct for further use, a total of five micrograms of genomic DNA from pooled wild type and the Hermes reporter line, pHer[A5C-EGFP], were analyzed by Southern blot. Both samples were digested with either HindIII or XbaI (New England Biolabs, Ipswitch, MA) according to the manufacturer, and incubated overnight at $37^{\circ} \mathrm{C}$. The digests were spiked the following morning and continued to digest for approximately $5 \mathrm{~h}$. Samples were fractionated on a $0.7 \%$ agarose/TAE gel overnight at $22 \mathrm{~V}$. The DNA was transferred and hybridized as previously described in (Smith et al. 2007).

An approximately 400 base pair DNA probe corresponding to the Hermes right end was prepared by digesting the pBSHerLR plasmid (Laver and Atkinson, unpublished) with $K p n I$ and $X h o I$ for $3 \mathrm{~h}$ at $37^{\circ} \mathrm{C}$. The sample was gel purified with the Zymoclean ${ }^{\mathrm{TM}}$ Gel DNA Recovery kit (Zymo Research, Orange, CA) and radiolabeled with the Prime-It ${ }^{\circledR}$ Random Primer Labeling kit (Stratagene, Cedar Creek, TX). Labeled probe was subsequently purified with a Micro Bio-Spin 30 Column (BioRad, Hercules, CA), boiled for $5 \mathrm{~min}$ then added to the hybridization solution. The Hybond-N ${ }^{\circledR}$ positively charged nylon membrane (Amersham Pharmacia, Buckinghamshire, England) was hybridized overnight at $65^{\circ} \mathrm{C}$, and then subsequently washed with $2 \times \mathrm{SSC} / 0.1 \% \mathrm{SDS}$ and $0.1 \times \mathrm{SSC} / 0.1 \% \mathrm{SDS}$ at room temperature for $15 \mathrm{~min}$ each wash. A more stringent wash with $0.1 \times \mathrm{SSC} / 0.1 \% \mathrm{SDS}$ was done at $65^{\circ} \mathrm{C}$ for $20 \mathrm{~min}$, and 
then exposed to Kodak Biomax MS film with an intensifying screen for approximately $20 \mathrm{~h}$ at $-80^{\circ} \mathrm{C}$.

Individual mosquitoes from reporter/helper crosses were analyzed by digesting half mosquito equivalents with HindIII (NEB) and incubated overnight at $37^{\circ} \mathrm{C}$, then spiked the following morning and continued to digest at $37^{\circ} \mathrm{C}$ for approximately five more hours. DNA was fractionated, transferred, and hybridized as mentioned above. Membranes were washed with $2 \times \mathrm{SSC} / 0.1 \% \mathrm{SDS}$ and $0.1 \times \mathrm{SSC} / 0.1 \% \mathrm{SDS}$ at room temperature for $15 \mathrm{~min}$ each wash, then more stringently with $0.1 \times \mathrm{SSC} / 0.1 \% \mathrm{SDS}$ at $65^{\circ} \mathrm{C}$ for $5 \mathrm{~min}$. Membranes were then exposed to Kodak Biomax MS film for approximately $48 \mathrm{~h}$ at $-80^{\circ} \mathrm{C}$.

Transposable element display analysis

Transposable element (TE) display was performed as previously described, with slight modification (Guimond et al. 2003; Wilson et al. 2003). Genomic DNA was isolated from individual mosquito samples resulting from the Hermes reporter/helper crosses using the Wizard ${ }^{\circledR}$ Genomic DNA Purification kit (Promega, Madison, WI). Approximately $100 \mathrm{ng}$ from each sample was digested with $\mathrm{Msp}$ I according to the reaction conditions supplied by the manufacturer (New England Biolabs, Ipswitch, MA). Following the digests, 60 pmol of adapters were ligated to the digested DNA and incubated overnight at $37^{\circ} \mathrm{C}$. The adapter consists of two oligonucleotide primers annealed to each other to create a "sticky ended" double stranded DNA product designed to ligate to a $M s p I$ half site. The $M s p$ I adapter also consists of the MseIA new (5'-GGATCCTACGACGATG AGTCCTGAG- $3^{\prime}$ ) and of the MspIB new (5'-CGCTCAGG ACTCATCGTCGTAGGATCC-3') oligonucleotides. Primary PCR reactions were carried out using a $1 / 50^{\text {th }}$ ligation reaction volume to serve as a DNA template.

The left end of the Hermes element integrations were analyzed by an initial PCR reaction assembled with a primer pair consisting of the MseIA new and Hermes LE 169R (5'-CAAGTAACAACACTTGATGTGCTGAGAGC-3'). Primary PCR products were diluted 10 times with $0.1 \times \mathrm{TE}$ buffer, then a $1 / 80^{\text {th }}$ reaction volume served as a template for the secondary reaction. A second, selective PCR reaction was performed using a $\gamma-{ }^{32} \mathrm{P}$ end labeled primer to detect specific bands of interest using a nested Hermes LE 108 R primer (5'-AGAACGACACGACAAAACATACCCGAG$\left.3^{\prime}\right)$ and MseIA new. The analysis of the Hermes right end integrations were carried out in a primary $\mathrm{PCR}$ reaction with the MseIA new and Hermes RE 155F (5'-GTTTGTA CACTTTTTACTCTCATACTCTAGCGGTG-3') primers. After amplification, samples were diluted and nested secondary reactions were done with the MseIA new and radiolabeled Hermes RE 95F (5'-CTGTTGTTGTTAAGTTGTG AAAAAATACTTCGTG-3') primers.
Prior to size fractionation on a $5 \%$ denaturing polyacrylamide gel (1.2× TBE buffer, 5\% Long Ranger (Fisher Scientific, Pittsburgh, PA), 7 M urea), loading buffer (96\% formamide, $20 \mathrm{mM}$ EDTA, $0.05 \%$ xylene cyanole FF, $0.05 \%$ bromophenol blue) was added to the secondary PCR products for each sample and heated at $65^{\circ} \mathrm{C}$. Electrophoresis was performed at constant power (50 watts) for approximately $2 \mathrm{~h}$, transferred to $3 \mathrm{MM}$ paper and vacuum dried. TE display gels were visualized after exposure to Kodak Biomax MS film at $-80^{\circ} \mathrm{C}$ for variable time periods depending on the strength of signal. A $25 \mathrm{bp}$ ladder (Invitrogen, Carlsbad, CA) was used to determine molecular weight of the amplified fragments, after it was radiolabeled according to the manufacturer. Bands of interest were excised from the gel, placed in $200 \mu \mathrm{l}$ of $0.1 \times \mathrm{TE}$ buffer, and allowed to resuspend from $2 \mathrm{~h}$ to overnight.

Tertiary PCR amplifications with the resuspended excised gel samples as a template, were assembled with the MseIA new primer and an unlabeled nested primer for the Hermes left end (Hermes LE 66R 5'-GAATTTTTTGTTCAAGTGGCAAAGCAC-3') or right end (Hermes RE 60F 5'-AAATACTTGCACTCAAAAGGCTTGACACC-3'). To remove non-specific PCR products, only tertiary PCR samples that appeared to be nested products of the secondary reaction were excised and gel purified with the Zymoclean $^{\text {TM }}$ Gel DNA Recovery kit (Zymo Research, Orange, CA). Recovered products were then cloned into a pJET cloning vector (Fermentas, Hanover, MD) and sequenced. Resultant sequence information was used to perform a BLAST search analysis for the Ae. aegypti genome using the VectorBase website (Lawson et al. 2007).

All PCR reactions were amplified with MasterTaq (Eppendorf, Hamburg, Germany) with the reaction parameters: 3 min initial denaturation at $95^{\circ} \mathrm{C}$, followed by five cycles in which the annealing temperature was decreased by one degree every cycle $\left(95^{\circ} \mathrm{C}\right.$ for $30 \mathrm{~s}, 68^{\circ} \mathrm{C}$ for $30 \mathrm{~s}$, and $72^{\circ} \mathrm{C}$ for one minute). A second cycling step of $95^{\circ} \mathrm{C}$ for $30 \mathrm{~s}, 65^{\circ} \mathrm{C}$ for $30 \mathrm{~s}$, and $72^{\circ} \mathrm{C}$ for one minute was repeated for 25 cycles, with a final extension step of $72^{\circ} \mathrm{C}$ for five minutes.

\section{Results}

Construction of transgenic Ae. aegypti helper/reporter lines

Transgenic "helper" lines of Ae. aegypti were created using the M3DB2Her construct consisting of a Mosl transposable element that contains a 3xP3-DsRed cassette as a marker for integration, and Hermes transposase under the control of the testis-specific Ae. aegypti $\beta 2$ tubulin promoter (Smith et al. 2007) (Fig. 1). A total of 349 


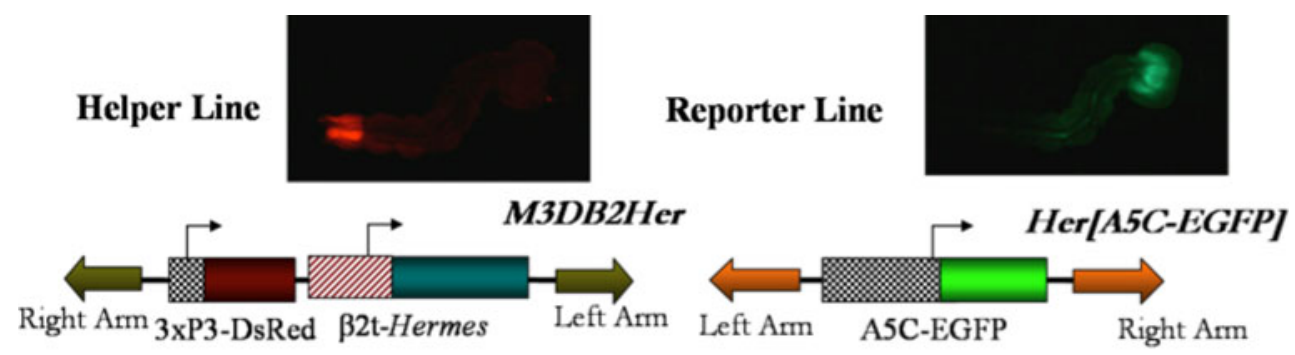

$\mathrm{X}$
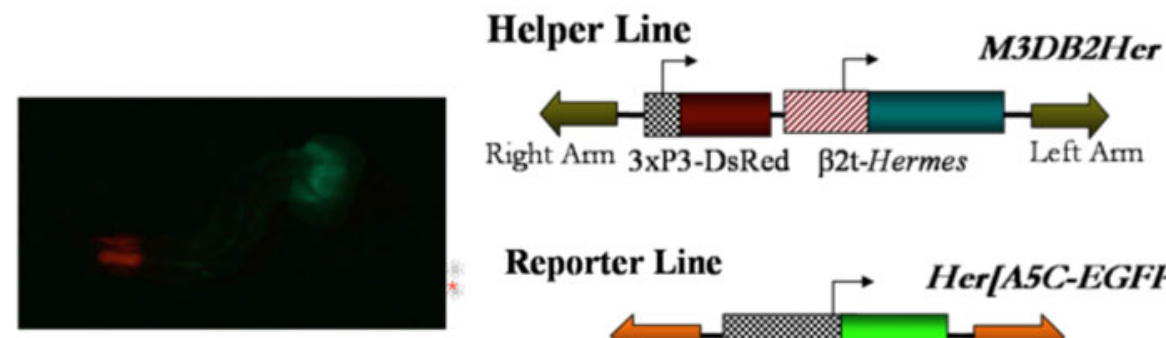

\section{Reporter Line}

Fig. 1 Schematic diagram of the reporter and helper lines used to examine Hermes mobility. A helper transgenic line, M3DB2Her, was established with a Mos 1 element containing the Hermes ORF under the regulation of $\sim 1 \mathrm{~kb}$ of the $A a \beta 2 \mathrm{t}$ promoter, with a $3 \times \mathrm{PP} 3$-DsRed cassette serving as a marker of integration. This was then crossed to the pre-existing Hermes element reporter line, pHer[A5C-EGFP], containing the actin5C promoter regulating expression of EGFP as established by Pinkerton et al. (2000). Heterozygous individuals with

embryos were injected, producing 76 surviving adults that resulted in two independent transgenic lines. Both lines were determined to be the result of a single integration by Southern blot, and were integrated into the Ae. agypti genome by a standard cut-and-paste mechanism as determined by TE display analysis (data not shown). Using sequence information from the recovered PCR fragments, the \#12M helper line mapped to supercontig 431, but the precise genomic location of the \#3M helper line could not be determined due its integration into highly repeated DNA (data not shown).

The Hermes "reporter" line had been previously established (Pinkerton et al. 2000) and consists of a Hermes element containing an EGFP fluorescent marker under the control of the D. melanogaster actin 5C promoter (pHer[A5C-EGFP]) (Figs. 1, 2a). The non-canonical nature of this integration was previously described, but further delineated by TE display analysis to determine the amount of plasmid DNA incorporated with the integration. On the left end of the integration 996 base pairs of plasmid were found to be incorporated, as well as greater than 310 base pairs of right end flanking plasmid (Fig. 2c). Unfortunately, due to the location of a MspI site used for the TE display digests at the left end breakpoint into Ae. aegypti

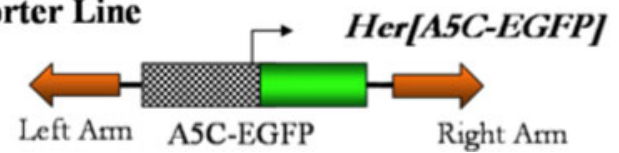

both the helper and reporter lines were selected by their respective genetic markers after the helper and reporter lines were crossed. Individuals with both markers were then crossed to wild type individuals or self-crossed to heterozygote siblings to examine for Hermes mobility. $\mathrm{EGFP}^{+}$progeny were then used for subsequent analysis. Asterisk indicates that this an overlayed image of a single larvae visualized under the appropriate EGFP and DsRed filters

genomic DNA, the genetic locus of the integration could not be determined. The pHer[A5C-EGFP] line was found to be heterologous in the number of Hermes element insertions by Southern blot analysis (Fig. 2b). A second insertion was detected in a portion of the pooled samples, inferred by the weak intensity of the band in Fig. 2b, and was later verified through individual mosquito Southern blots. It is unclear whether this second insertion was the result of a second integration previously undetected in the original transformation experiment or the result of a subsequent recombination event.

\section{Expression of Hermes transposase}

The expression of Hermes transposase was determined through the use of RT-PCR for both M3DB2Her helper lines (Fig. 3). For both helper lines transposase expression was weak, but was unique in the pattern of expression between the two lines. Transposase expression in the \#3M helper line was male specific in pupal and adult samples, but did not appear to be tightly confined to the germline. Within the \#12M helper line, transposase was only expressed in the male and female adult samples, with higher levels of expression present in adult males. In both transgenic lines, 
A

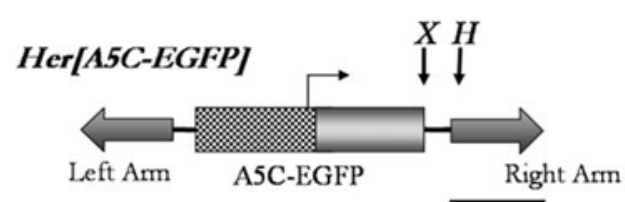

C
B

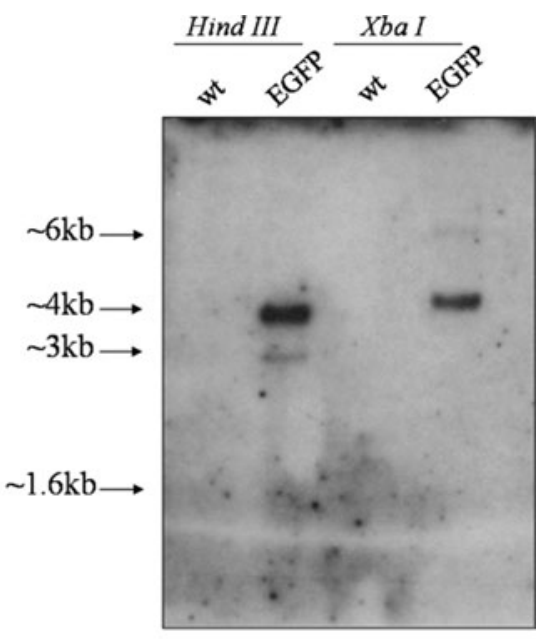

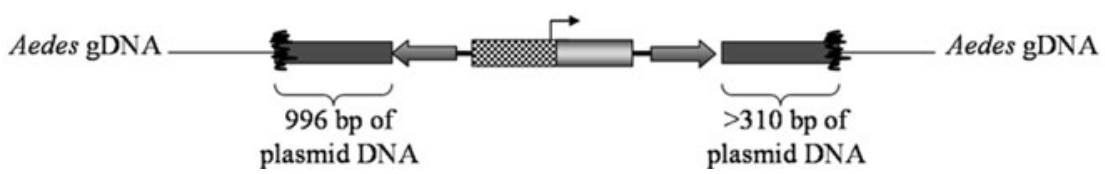

Fig. 2 Integration analysis of the Ae. aegypti pHer[A5C-EGFP] reporter line. a Schematic map of the pHer[A5C-EGFP] construct containing the D. melanogaster actin 5C (A5C) promoter regulating EGFP expression. The black bar represents the $\sim 400$ base pair right arm fragment probe used in the Southern blotting analysis. The locations of restriction sites within the construct are indicated by $\mathrm{X}$ (XbaI) and $\mathrm{H}$ (HindIII). b Southern blot analysis of the previously established pHer[A5C-EGFP] reporter line and wild type pooled DNA after digestion with either HindIII or and XbaI. Two bands were detected in both digests of the transgenic EGFP line with variable intensity, indicative of a heterogeneous pooled sample of either one or two Hermes integrations. No bands were detected in the wild type DNA. Sizes of detected bands are indicated on the left. c To better define the integration of the major parental integration from Pinkerton et al. (2000), TEDA was used to determine the breakpoint junctions between flanking plasmid DNA and the integration into the Ae. aegypti genome. Although the genomic location could not be determined, 996 base pair of plasmid sequence was found to flank the left end integration, and greater than 310 base pairs of plasmid sequence on the right end
Hermes transcript was detected at its highest levels in the testes, but was also present within the dissected male carcass. This apparent loss of tissue-specificity has also been detected in other studies of the Aedes $\beta 2$ tubulin promoter (R. C. Smith and P. W. Atkinson, unpublished) and suggests that the Aedes $\beta 2$ tubulin promoter may be affected by position effects associated with the integrations of the element. Position effects have been previously shown to influence the expression of a reporter in transgenic lines of Ae. aegypti (Coates et al. 1998, 1999; Jasinskiene et al. 1998), and if inserted into a heterochromatic region of the genome, could explain the low levels of Hermes transcript produced from the helper construct.

\section{Analysis of Hermes remobilization}

To test the ability of a Hermes element to remobilize in the germline of Ae. aegypti, heterozygous jumpstarter lines resulting from the mating of a Hermes element reporter line and a Hermes transposase producing helper line were selected by the simultaneous presence of DsRed and EGFP markers (Fig. 1). For both transgenic helper lines, male and female helper/reporter mosquitoes were isolated and crossed to wild type individuals. Self crosses of helper/ reporter individuals were also conducted for both helper lines (Table 1). For each helper line duplicate crosses were conducted and referred to as $\Delta$ and $\Gamma$ for the \#3M helper line, and $\Sigma$ and $\Phi$ for the \#12M helper line. Progeny were screened for the inheritance of each marker, with $\mathrm{EGFP}^{+}$ progeny containing the Hermes element collected for further analysis. The inheritance of both fluorescent genetic markers occurred in approximately $25 \%$ of the progeny examined, suggesting that both helper line integrations are located on separate chromosomes from the Hermes reporter integration.

\section{Analysis of $\mathrm{EGFP}^{+}$progeny by Southern blot}

Progeny collected from reporter/helper crosses containing a Hermes element were analyzed by Southern blots to determine the presence of any germline remobilization events. A total of 1,491 individual $\mathrm{EGFP}^{+}$mosquito samples resulting from crosses of both helper lines were examined. A total of 787 individual $\mathrm{EGFP}^{+}$progeny from the \#3M helper line (crosses $\Delta$ and $\Gamma$ ) and 704 individual $\mathrm{EGFP}^{+}$progeny from $\# 12 \mathrm{M}$ helper line $(\Sigma$ and $\Phi)$ were 


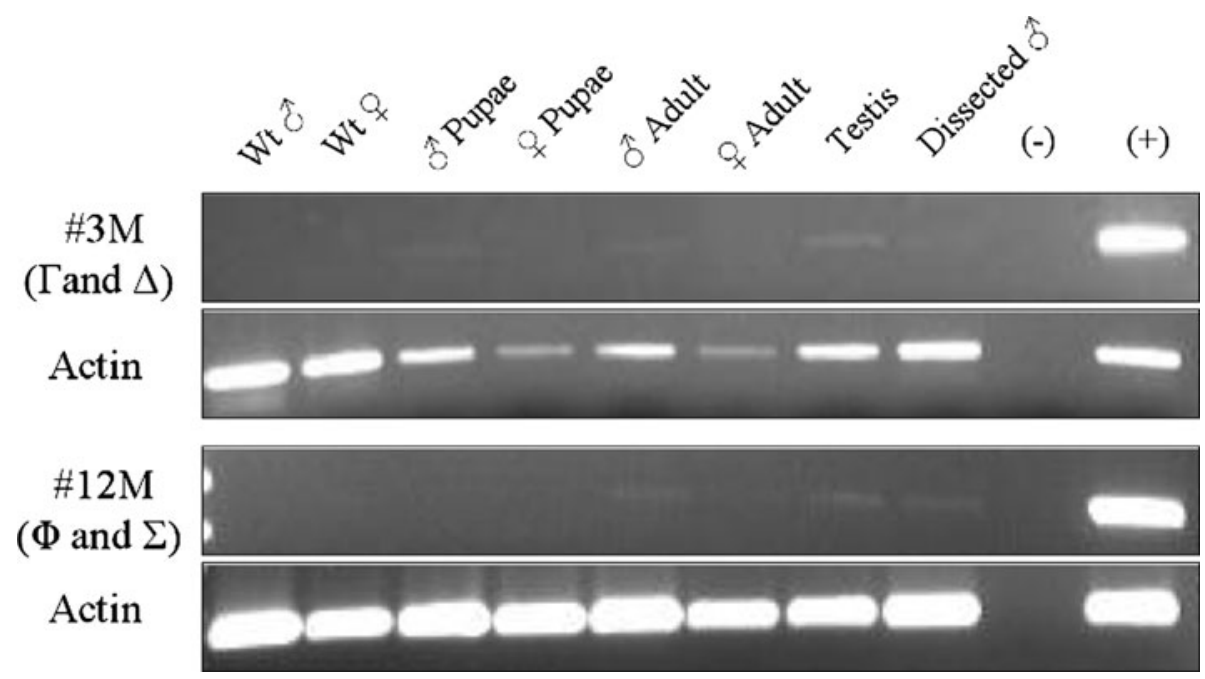

Fig. 3 Expression of Hermes transposase in transgenic helper lines. Mosquitoes were collected at various stages of development to isolate RNA from the \#3M (used to set up the $\Delta$ and $\Gamma$ crosses) and \#12M (used to set up the $\Sigma$ and $\Phi$ crosses) helper lines. RNA was also isolated from the testes and the dissected male carcass from both helper lines. RT-PCR was used to analyze Hermes transposase

Table 1 Summary of Hermes helper/reporter crosses

\begin{tabular}{|c|c|c|}
\hline Cross & $\begin{array}{l}\mathrm{EGFP}^{+} \text {progeny } \\
\text { collected }\end{array}$ & $\begin{array}{l}\text { Progeny } \\
\text { analyzed }\end{array}$ \\
\hline$\# 3 \mathrm{M} / \mathrm{EGFP} \hat{\delta} \times \mathrm{wt}$ 우 & 320 & 320 \\
\hline 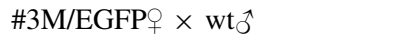 & 230 & 230 \\
\hline$\# 3 \mathrm{M} / \mathrm{EGFP} \hat{\delta} \times \# 3 \mathrm{M} / \mathrm{EGFP}+$ & 328 & 237 \\
\hline \#3M Total & & 787 \\
\hline$\# 12 \mathrm{M} / \mathrm{EGFP} \hat{\delta} \times \mathrm{wt}^{\circ}$ & 254 & 254 \\
\hline$\# 12 \mathrm{M} / \mathrm{EGFP} \propto \times \mathrm{wt}^{\circ} \hat{\sigma}$ & 296 & 296 \\
\hline$\# 12 \mathrm{M} / \mathrm{EGFP}_{\widehat{o}} \times \# 12 \mathrm{M} / \mathrm{EGFP}_{+}$ & 197 & 154 \\
\hline \#12 Total & & 704 \\
\hline Total & & 1,491 \\
\hline
\end{tabular}

Hermes remobilization in the germ line of Ae. aegypti was analyzed through progeny of reporter/helper crosses containing a Hermesproducing helper line (\#3M or \#12M) and a Hermes element. Individual reporter/helper mosquitoes expressing transposase, with either helper line, were mated to wild type or self crossed individuals. Progeny were collected from $\mathrm{EGFP}^{+}$individuals and analyzed by Southern blot. Samples that resulted in a new putative integration were subsequently analyzed by TE display

analyzed by Southern blot (Table 1). Several progeny from both helper line crosses were identified as containing putative transpositions based upon the detection of unique non-parental bands, which were primarily identified as the result of germline or early somatic movement. Furthermore some containing an additional band did not contain the parental band, indicative of an excision event (Fig. 4a, b). In very few cases there was an absence of any band and, given that these progeny were selected based on expression in transgenic M3DB2Her lines PCR, and compared to nontransgenic (wild type male and female) samples using 28 cycles of PCR. Cytoplasmic actin levels were used to normalize between samples. A non-template reaction (-), and genomic DNA from the helper lines (+) were used as PCR controls

fluorescence phenotype, we interpreted this as being most likely due to sample loss.

Within the \#3M helper line samples, very little evidence of new transposition products were detected in the male and female outcrosses. Evidence of early somatic transposition (based upon molar ratios in comparison to the parental band) were found in one out of the 320 male outcross progeny examined, and two individuals from the 230 female outcross progeny (illustrated by $Г 3 \mathrm{~F} \# 22$ in Fig. 4e). No apparent germline movement was detected in any outcross samples of the \#3M helper line. Of the 237 progeny examined resulting from a self-cross of helper and reporter parents, twelve unique progeny were detected containing new integrations that are believed be of either early somatic or germline in origin (Fig. 4a).

A total of seven unique putative germ line or early somatic events were detected in the male outcross samples from the \#12M helper line (Fig. 4b, c). However, a subset appeared to result from a partial transposition or recombination event. After originally identified as putative germline transpositions upon Southern blot hybridization to the right end (Fig. 4c), re-probing to the Hermes left end failed to produce any non-parental bands (Fig. 4d).

One unique event was detected in four of 38 progeny resulting from a female outcross of the \#12M helper line, $\Phi 1 \mathrm{~F}$ (Fig. 4e). The 258 progeny from the other female cohorts analyzed did not produce any evidence of transposition (Fig. 4e). No evidence of re-mobilization was detected in the 154 progeny from the \#12M helper selfcrosses (data not shown). 

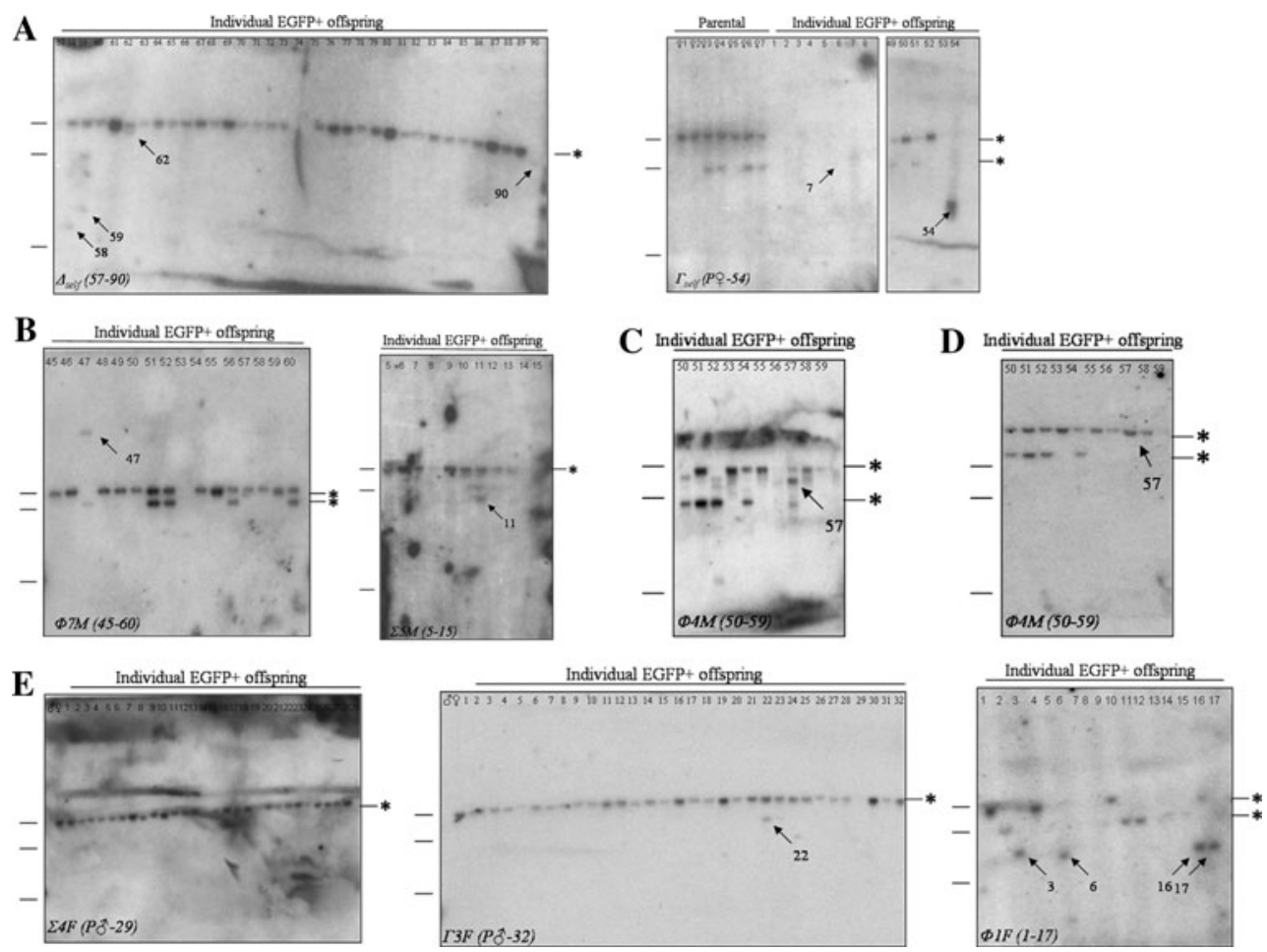

Fig. 4 Southern blot analysis of progeny resulting from reporter/ helper jumpstarter crosses for the Hermes element. Genomic DNA from individual $\mathrm{EGFP}^{+}$samples were digested with HindIII and probed with a $\sim 400 \mathrm{bp}$ Hermes right end fragment as in Fig. 3. Individual progeny from the self crosses $\left(\beta 2 \mathrm{t}\right.$-Hermes $/ \mathrm{EGFP}^{+} \hat{\sigma} \times \beta 2 \mathrm{t}$-Hermes $/$

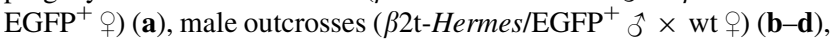
and female outcrosses (wt $\hat{\sigma} \times \beta 2 \mathrm{t}$-Hermes $/ \mathrm{EGFP}^{+}$क) (e) were analyzed. In some instances, as evidenced by the $\Phi 4 \mathrm{M}$ cross $(\mathbf{c}, \mathbf{d})$, the resultant Southern blot patterns did not correlate when probed with fragments from the Hermes right (c) or left ends (d). Heterogeneity for

\section{Analysis of exceptional $\mathrm{EGFP}^{+}$progeny by TE display}

Exceptional progeny resulting from the Southern blot analysis thought to contain putative germline or early somatic remobilization events were further analyzed by TE display to determine the sequences associated with the new integrations for the left and right ends of the Hermes element.

Due to the large amount of flanking plasmid surrounding the parental pHer[A5C-EGFP] integration (Fig. 2c), TE display analysis proved difficult since the length of a PCR product for the left end integration is $\sim 1.1 \mathrm{~kb}$ (Fig. 5a) and the right end integration was undefined. As a result, very few of the samples that underwent TE display yielded sequence information. Furthermore, incomplete PCR products were detected that did not contain the proper restriction enzyme half site after the ligation of adapter sequences, possibly due to star activity of the $M s p I$ enzyme during the overnight digest. Consequently, several of the recovered PCR products contained an intact left or right end sequence followed by a length of DNA before an improper half site and ligated adapter sequence to produce a the number of Hermes copies was found within the parental EGFP ${ }^{+}$line with either one or two insertions present at $\sim 3$ or $4 \mathrm{~kb}$. For each Southern blot the parental integrations were marked by an asterisk. Three bars, corresponding to $\sim 1.6,3$, and $4 \mathrm{~kb}$ are denoted respectively for each Southern blot. The samples that were examined are denoted in italics for each cross and the corresponding progeny that were analyzed. Exceptional progeny with new integrations based on Southern blot analysis are marked by an arrow and labeled by sample number

specific product. It is possible that these are PCR artifacts, but given that the Hermes ends and adapter sequences are intact, we believe that the recovered integrations are most likely incomplete products of authentic transposition events. Despite the problems associated with the noncanonical parental integration for analysis by TE display, this method was able to produce clearly defined new transposition products devoid of the parental element in some instances (Fig. 5b).

Of the events recovered for the Hermes left and right ends (Tables 2, 3), integrations fall into one of three categories: precise cut-and-paste events, non-canonical cutand-paste events involving flanking plasmid sequence, and non-canonical cut-and-paste events involving deletions of the Hermes ends. All of the precise cut-and-paste events recovered were assigned as somatic events based on the propensity for somatic transposition demonstrated by the previous behavior of Hermes within mosquitoes (O'Brochta et al. 2003). This is supported through experimental evidence with the $\Delta_{\text {self }} \# 59$ and \#62 samples that produced a new putative band in Southern blot analysis that was 
Fig. 5 Examination of exceptional progeny from jumpstarter lines by TE display analysis. a Progeny containing the $\sim 1.1 \mathrm{~kb}$ parental band integration were analyzed by TEDA, with sizes marked by a 25 bp ladder. Other bands present are non-specific products of the PCR reaction, as determined by gel excision, further amplification, and cloning. b Two of the exceptional progeny, as determined by Southern blot analysis, were verified by TEDA displaying new single germline integrations. New bands are demonstrated by the corresponding arrows

A

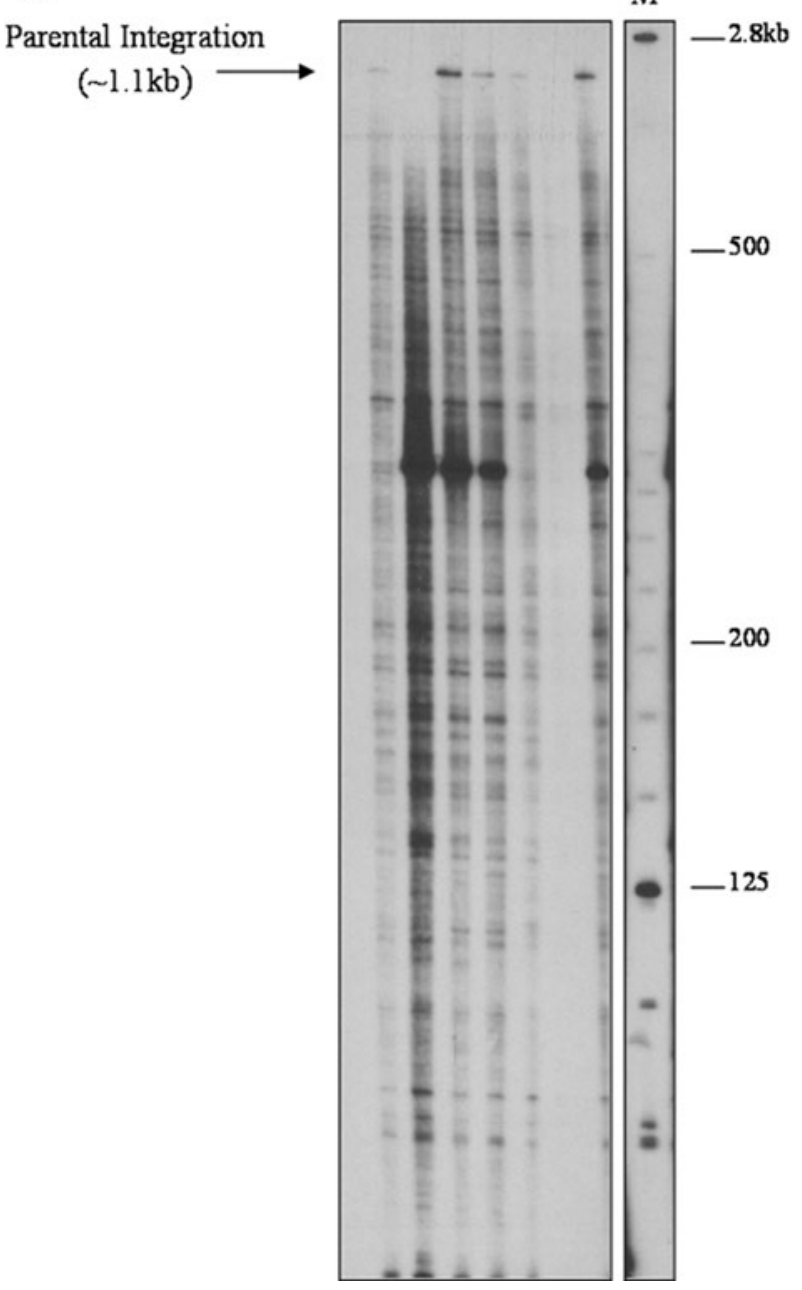

B
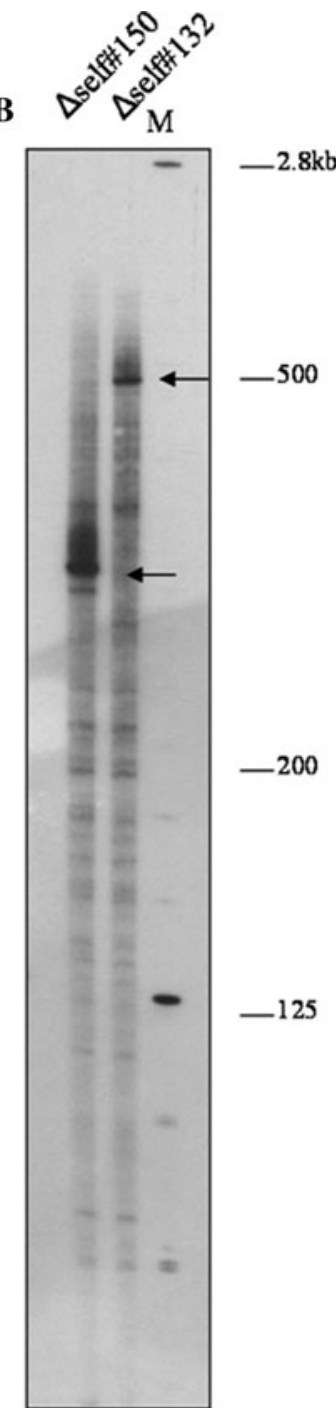

non-equimolar to the parental integration (Fig. 4a), and a cut-and-paste transposition event for the Hermes left end upon TE display analysis (Table 2).

In eight integrations, flanking plasmid was integrated along with the element reminiscent of previously described integrations of Hermes within mosquitoes (Allen et al. 2001; Jasinskiene et al. 2000; Pinkerton et al. 2000). However, since the remobilization strategy relies on an existing non-canonical integration (Pinkerton et al. 2000), these new integrations display breakpoints within the existing flanking plasmid sequence. Breakpoints were recovered on the left end plasmid flank at 71, 72, 95, 115, 192, 194, and $270 \mathrm{bp}$ from the terminal inverted repeats (TIR) on the left end of the element (Table 2) and at $117 \mathrm{bp}$ from the TIR on the right end of the element (Table 3) as summarized in Fig. 6a.

Other types of recovered integrations involved internal deletions of the left and right arms of Hermes, including the regions corresponding to the TIR of the element.
Deletions of $2,8,13,17,18,22$, and 54 bp were detected for the Hermes left end (Table 2) and of 24, 42, 52, 56, and $60 \mathrm{bp}$ for the right end (Table 3 ).

\section{Discussion}

The use of transposable elements as mutagens to identify gene and promoters based on function and population replacement strategies in mosquitoes are heavily reliant on high rates of post-integration transposition. Despite the promise of such strategies, previous work has demonstrated that integrated transgenic transposons display very little or no remobilization within the mosquito germline (O'Brochta et al. 2003; Scali et al. 2007; Sethuraman et al. 2007). In an effort to overcome this significant hurdle, transgenic lines that express Hermes transposase under the control of the $A a \beta 2 \mathrm{t}$ promoter within the male germline of $A e$. aegypti were created to analyze the rate of Hermes 
Table 2 Summary of Hermes left end integrations

\begin{tabular}{|c|c|c|c|}
\hline Integration site & Left end & Sample & Genomic locus \\
\hline (breakpoint after 95 bp of plasmid flank) & CAGAGAACAACAACAAG TGGCTTATTTTGAT & $\Delta$ self \#1 & Superconting $729^{\mathrm{a}}$ \\
\hline ...GCCGATTGGGCTTTCATTTACGGAGAATTTT & $C A G A G A A C A A C A A C A A G$ TGGCTTATTTTGAT & $\Delta$ self \#1 & DmA5C promoter \\
\hline (breakpoint after 194 bp of plasmid flank) & $C A G A G A A C A A C A A C A A G$ TGGCTTATTTTGAT & $\Delta$ self \#1 & DmA5C promoter ${ }^{\mathrm{a}}$ \\
\hline$\underline{\mathrm{C}}$ & GGCTTATTTTGAT & $\Delta$ self \#14 & $\mathrm{NS}^{\mathrm{a}}$ \\
\hline ...GACCACTAAGAAACTAGATATGTGTACCAAT & $C A G A G A A C A A C A A C A A G$ TGGCTTATTTTGAT & $\Delta$ self $\# 59$ & Superconting $130^{\mathrm{b}}$ \\
\hline (breakpoint after 192 bp of plasmid flank) & $C A G A G A A C A A C A A C A A G$ TGGCTTATTTTGAT & $\Delta$ self $\# 132$ & ND \\
\hline (breakpoint after 72 bp of plasmid flank) & $C A G A G A A C A A C A A C A A G$ TGGCTTATTTTGAT & $\Delta$ self $\# 150$ & Superconting 335 \\
\hline (breakpoint after $270 \mathrm{bp}$ of plasmid flank) & $C A G A G A A C A A C A A C A A G$ TGGCTTATTTTGAT & Гself \#7 & Superconting $335^{\mathrm{b}}$ \\
\hline ...CGAGGCACTGGCTGAAATTGGTTTTGTCTG & $C A G A G A A C A A C A A C A A G$ TGGCTTATTTTGAT & $\Gamma$ self \#54 & NS \\
\hline ...CTTTGTATCTGCTGACGATGGGCCTCAAAG & $A A C A A C A A G$ TGGCTTATTTTGAT & $\Gamma$ self \#54 & NS \\
\hline (breakpoint after $71 \mathrm{bp}$ of plasmid flank) & $C A G A G A A C A A C A A C A A G$ TGGCTTATTTTGAT & $\Gamma$ self \#54 & Superconting 151 \\
\hline ...TAGAAATTCCTGCTAAACTGCTGCAAGGAT & $C A G A G A A C A A C A A C A A G$ TGGCTTATTTTGAT & $\Sigma 5 \mathrm{M} \# 6$ & NS \\
\hline ...TGAAATGTCAACACATATTTCGCTTAAAAC & (deletion of $54 \mathrm{bp}$ ) & $\Sigma 5 \mathrm{M} \# 11$ & Superconting $203^{\mathrm{a}}$ \\
\hline ...AATAACTAAGACAAACCAACTNTGAATCAA & ATTTTGAT & $\Sigma 5 \mathrm{M} \# 11$ & Superconting $500^{\mathrm{a}}$ \\
\hline ...TCCGAGTGCTACAAAAAGTATCAAGAGTGG & GAGAACAACAACAAGTGGCTTATTTTGAT & $\Sigma 5 \mathrm{M} \# 15$ & Superconting $551^{\mathrm{a}}$ \\
\hline ...GTTTGTTCAAAGTTGTCTTGGAAGGTCTCT & CAAGTGGCTTATTTTGAT & $\Sigma 5 \mathrm{M} \# 15$ & $\mathrm{ND}^{\mathrm{b}}$ \\
\hline ...AGGTTGACGTGTTTCTTTCTTCTTCTGTTC & TGGCTTATTTTGAT & $\Sigma 5 \mathrm{M} \# 15$ & $\mathrm{ND}^{\mathrm{b}}$ \\
\hline ...AGCAGCACTACGATGAACACCTGAATGGCG & $C A G A G A A C A A C A A C A A G$ TGGCTTATTTTGAT & $\Sigma 5 \mathrm{M} \# 15$ & Superconting $827^{\mathrm{b}}$ \\
\hline (breakpoint after 115 bp of plasmid flank) & $C A G A G A A C A A C A A C A A G$ TGGCTTATTTTGAT & $\Phi 2 \mathrm{M} \# 47$ & ND \\
\hline
\end{tabular}

New pHer[A5C-EGFP] integrations resulting from the reporter/helper crosses are represented by the 17 bp of the left (5') ITR (in italics) and adjoining sequences flanking the element for each sample, with genomic positions within Ae. aegypti represented by Supercontig number. Deletions of the ITR are shown in respect to the full length $17 \mathrm{bp}$ ITR, or by parenthesis. Underlined bases correspond to the restriction half site before immediately followed by adapter sequence. NS, not specific BLAST. ND, the exact genetic loci could not be determined

${ }^{a} M s p I$ half site could only be identified in a portion of clones recovered

${ }^{\mathrm{b}}$ MspI half site was not found in any of the clones recovered

Table 3 Summary of Hermes right end integrations

\begin{tabular}{|c|c|c|c|}
\hline Right end & Integration site & Sample & Genomic locus \\
\hline ACGTTTGCCTGTGACTTGTTGAAGTTCTCTG & ACCG & $\Delta$ self \#1 & $\mathrm{NS}^{\mathrm{a}}$ \\
\hline (deletion of $52 \mathrm{bp}$ ) & ATGCAGCACGGCATCCTCTCGTACCG & $\Delta$ self \#6 & Superconting $96^{\mathrm{a}}$ \\
\hline ACGTTTG & GGGCTGGCATCTCGCCAGAGCACTGCGCAAC... & $\Delta$ self \#14 & ND \\
\hline ACGTTTGCCTGTGACTTGTTGAAGTTCTCTG & GTCTGTATAGATCTTCAAATCACTAGTGAATTC... & $\Delta$ self \#42 & $\mathrm{NS}^{\mathrm{a}}$ \\
\hline ACGTTTGCCTGTGACTTGTTGAAGTTCTCTG & GAGATATTTCGAAAAAAAATCAGGACGAACTA... & $\Delta$ self $\# 59$ & ND \\
\hline ACGTTTGCCTGTGACTTGTTGAAGTTCTCTG & CATCGGCGACGGCCCCGTGCTGCTGCCCGACA... & $\Delta$ self \#62 & NS \\
\hline ACGTTTGCCTGTGACTTGTTGAAGTTCTCTG & (breakpoint after $117 \mathrm{bp}$ of flanking plasmid) & $\Delta$ self $\# 90$ & Within parental $\mathrm{LE}^{\mathrm{b}}$ \\
\hline (deletion of $42 \mathrm{bp}$ ) & $\underline{\mathrm{CCG}}$ & $\Delta$ self $\# 90$ & NS \\
\hline (deletion of $60 \mathrm{bp}$ ) & AACCCCCTAGATTTCCG & $\Gamma$ self \#54 & NS \\
\hline ACGTTTGCCTGTGACTTGTTGAAGTTCTCTG & GTGGAGGCTAACAGTCGCCACAACCTCAGACA... & $\Sigma 5 \mathrm{M} \# 10$ & Superconting 1039 \\
\hline (deletion of $56 \mathrm{bp}$ ) & TGTTGACAAAACCACATTGCCTCGTTATGGAT... & $\Sigma 5 \mathrm{M} \# 11$ & Superconting 271 \\
\hline
\end{tabular}

New pHer[A5C-EGFP] integrations resulting from the reporter/helper crosses are represented by the 17 bp of the right $\left(3^{\prime}\right)$ ITR (in italics) and adjoining sequences flanking the element for each sample, with genomic positions within Ae. aegypti represented by Supercontig number. Deletions of the ITR are shown in respect to the full length $17 \mathrm{bp} \mathrm{ITR,} \mathrm{or} \mathrm{by} \mathrm{parenthesis.} \mathrm{Underlined} \mathrm{bases} \mathrm{correspond} \mathrm{to} \mathrm{the} \mathrm{restriction} \mathrm{half} \mathrm{site}$ before immediately followed by adapter sequence. NS, not specific BLAST. ND, the exact genetic loci could not be determined

${ }^{a} M s p$ I half site could only be identified in a portion of clones recovered

b The integration site occurred within the parental element, with the right end sequence transitioning into the last $78 \mathrm{bp}$ of the $\sim 1 \mathrm{~kb}$ plasmid sequence flanking the parental left end integration 


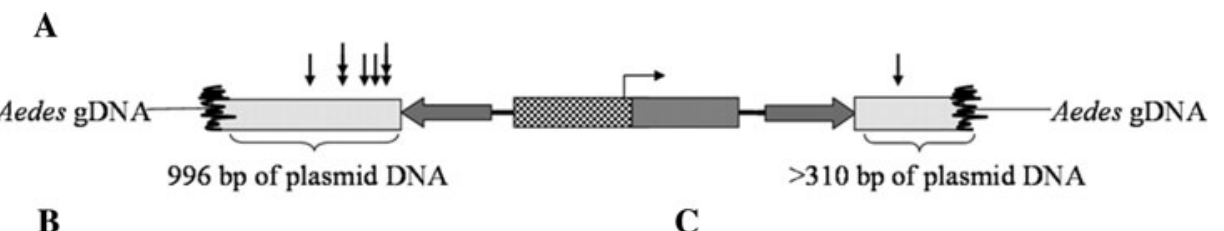

B

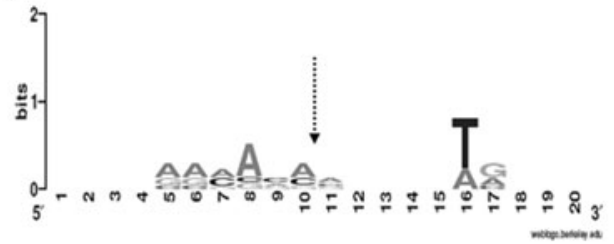

C

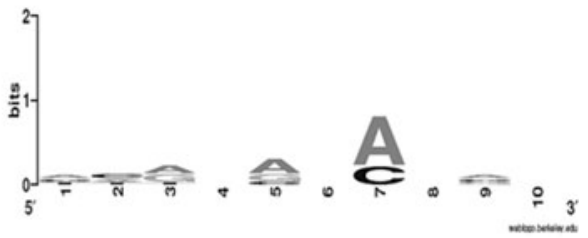

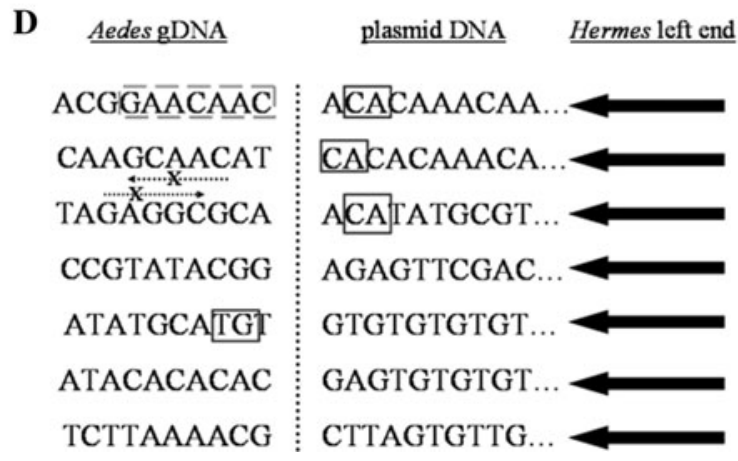

Fig. 6 Analysis of non-canonical event breakpoints in flanking plasmid DNA. a The locations of the recovered non-canonical event breakpoints are illustrated in correlation to the parental Hermes integration by arrows (map not drawn to scale). Recovered events that were localized to similar positions of the flanking plasmid sequence are shown by the double arrows. Consensus sequences surrounding the breakpoint ( \pm 10 base pairs) in the flanking plasmid DNA are summarized in (b), with the broken arrow symbolizing the plasmid breakpoint. The consensus sites of DNA integration into Aedes genomic DNA were similarly analyzed using WebLogo software (http://weblogo.berkeley.edu) with base 10 as the first base flanking the integration (c). Note that the consensus does not match the Hermes consensus target site integration of $5^{\prime}$-nTnnnnAn- $3^{\prime}$. Three of the seven left end integrations contain sites similar to known functional regions of the Hermes element ( $\mathrm{T}$. Laver and P. W. Atkinson, unpublished data) (d). The sequences surrounding the integrations of plasmid DNA into Aedes chromosomes are displayed with the dashed vertical line marking the site of integration. The samples that contain a $5^{\prime}$-CA dinucleotide similar to the terminal dinucleotides of the Hermes element responsible for cleavage are shown in boxes. Other regions of homology include an exact similarity to bases 5-11 of the Hermes left ITR (dashed box) and the presence of degenerate GTGGC binding motifs (dashed arrow with an X marking the site of degeneracy) in the flanking Aedes DNA. The remaining samples have no apparent homology

one or both ends of the Hermes element using TE display in 16 out of the 24 total samples. Among these recovered samples, two types of transposition were detected in the recovered sequences.

One type consisted of "cut-and-paste" transpositions which contained a Hermes element that has been precisely cleaved and inserted into the Ae. aegypti chromosomes, a behavior previously characterized to only occur during Hermes somatic transposition in Ae. aegypti (O'Brochta et al. 2003). For this reason, and supported by the nonequimolar bands detected in the Southern blot samples of $\Delta_{\text {self }} \# 59$ and \#62 (Fig. 4a), the cut-and-paste transpositions were assigned as being early somatic in origin. Although transposase expression was regulated by a testis-specific promoter, the presence of somatic events could be explained by three possible mechanisms. Based upon the RT-PCR results of both transgenic helper lines, Hermes transcripts were not tightly confined to the testes and were putative integrations, PCR fragments were isolated from 
found at detectable levels in the dissected male carcass and female samples (Fig. 2). Therefore, the recovered cut-andpaste events may be due to the expression of transposase within somatic tissue. Another explanation is supported by the prolonged presence of DsRed expression in transgenic sperm under the control of the $A a \beta 2 t$ promoter (Smith et al. 2007), which suggests that the stability of transposase expression after packaging into transgenic sperm could generate an early somatic transposition event upon fertilization. This is further supported by the detection of cutand-paste transposition by Southern blot analysis which would require transposition to occur at an early stage of development in order to be detected by hybridization. It is also conceivable that the somatic movement could also be attributed to the unknown identity of the second Hermes integration in the parental pHer[A5C-EGFP] reporter line. This second Hermes integration is not fixed within the maintained transgenic line and segregates normally within progeny as shown in Figs. 3 and 4. Therefore, it may have escaped initial detection and has subsisted in heterozygote form as the transgenic line has been maintained since its initial characterization (Pinkerton et al. 2000). Given this element should not contain a functional transposase gene, it is unclear how it could mediate the remobilization of the target element. Initial attempts to identify this second integration by PCR analysis were not conclusive (data not shown).

The second type of recovered fragments resulting from TE display were examples of the aberrant cut-and-paste behavior in which the Hermes element had been transposed with flanking plasmid DNA as previously demonstrated by Hermes integrations in the germline of Ae. aegypti (Jasinskiene et al. 2000; Pinkerton et al. 2000), or contained deletions internal to the element.

A total of eight events were recovered that displayed breakpoints within the flanking plasmid DNA surrounding the Hermes element upon their integration into the Ae. aegypti genome. Each of these new integrations displayed breakpoints different from that of the original parental integration, with the resulting insertions containing less plasmid flanking DNA. These products displayed breakpoints within the flanking plasmid at 71, 72, 95, 115, 192, 194, and $270 \mathrm{bp}$ from the terminal nucleotide of the Hermes left end, and at $117 \mathrm{bp}$ from the Hermes right end (summarized in Fig. 6a). Strikingly, breakpoints occur at similar positions between independent samples, suggesting a functional significance to these regions. Analysis of the bases in the immediate vicinity $(-10 /+10)$ of the breakpoints in the plasmid flanking sequence display only weak consensus at the cleavage site and within the newly formed end of the element (Fig. 6b). Upon transposition, it does not appear as though Hermes consensus eight base pair target site duplicated sequence is formed (Fig. 6c), and suggests that the non-canonical integrations arise as the result of a different mechanism than that of the traditional cut-and-paste integration containing a target site preference of nTnnnnAn (Sarkar et al. 1997). In four of the eight integrations, the breakpoints occur in flanking plasmid DNA that contain $5^{\prime} \mathrm{CA}$-dinucleotides at or near the site of cleavage (Fig. 6d). A similar 5' CA-dinucleotide is present at the terminal ends of the Hermes terminal inverted repeats (TIRs), and is the site of first strand cleavage (Zhou et al. 2004). Furthermore, in close proximity to the breakpoints are degenerate GTGGC motifs (Fig. 6d), a sequence that has demonstrated a strong binding capacity for Hermes transposase and is present in multiple copies on both ends of the element (T. Laver and P. W. Atkinson, unpublished data). Also present in one sample is an exact copy of bases 5-11 of the Hermes left TIR. Taken together, it would suggest that these non-canonical events leading to the integration of flanking plasmid sequence are transposase-mediated events that are the result of non-specific binding and cleavage events at Hermes-like sequences by Hermes transposase and not the result of random recombination events.

Six of the eight recovered fragments from five progeny (two fragments were recovered from the $\Delta_{\text {self }} \# 1$ sample) that also produced clearly defined non-parental bands in Southern blot analysis (some displayed in Fig. 4). The remaining progeny produced fragments indicative of germline events through TE display (Fig. 5). We therefore believe that the non-parental fragments recovered from these five progeny may be the result of Hermes re-mobilization events in the Ae. aegypti germline.

Other types of non-canonical events were also recovered in which deletions occurred within the left and right arms of the Hermes element. Deletions of the Hermes left end were recovered at $2,8,13,17,18,22$, and $54 \mathrm{bp}$ and at 24 , 42, 52, 56, and 60 bp from the Hermes right end (Fig. 7). For both ends of the element, it would appear as though the deletions are the result of an aberrant cleavage step during the transposition process. Normal cleavage of the Hermes element occurs at the cytosine residue present at position one within the TIR (Zhou et al. 2004), but based upon the locations of the deletions it would suggest that the nonspecific cleavage is occurring in close proximity to regions of transposase binding. The majority of the deletions for the Hermes left end occur within the first $30 \mathrm{bp}$ of the element where Hermes transposase has strong binding affinity to positions 1-5 bp and 14-23 bp (T. Laver and P. W. Atkinson, unpublished data). Also located within this region is a GTGGC binding motif located between 17 and $21 \mathrm{bp}$ of the left end, to which four of the seven deletion fragments are localized (Fig. 7a). The majority of the deletion fragments recovered for the Hermes right end also occur within regions with known binding for Hermes 


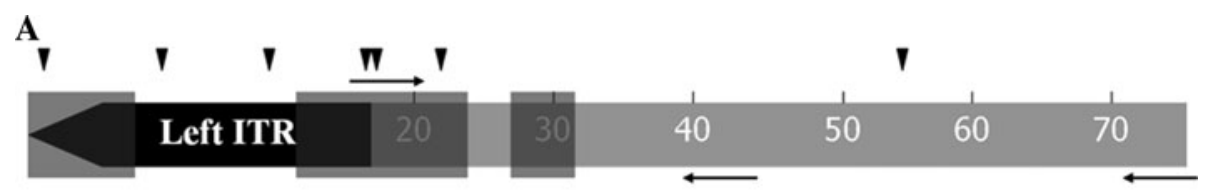

B

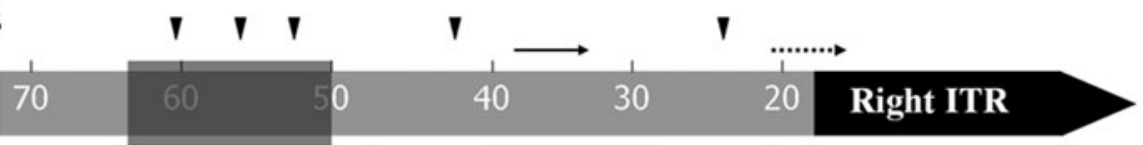

Fig. 7 Recovered deletion events within the Hermes element correlate to known regions of Hermes transposase binding. As a result of the TEDA analysis from the exceptional progeny obtained from Southern blot analysis, transposition events were recovered in which the ITRs and internal regions of the Hermes element were deleted upon integration into A. aegypti chromosomal DNA. The locations of these deletion breakpoints (arrowheads) are summarized for the Hermes left $\mathbf{a}$ and right ends $\mathbf{b}$. The 17 base pair ITRs are denoted in

transposase. Three of the five fragments recovered are contained within 64 to $50 \mathrm{bp}$ internal to the right end of the element, the location of the highest affinity binding by Hermes transposase for the right end (T. Laver and P. W. Atkinson, unpublished data). Deletion fragments were also recovered in close association to intact and degenerate GTGGC motifs present within the right end from 38 to 34 and 20 to $16 \mathrm{bp}$, respectively (Fig. 7b). However, it has been demonstrated that these regions do not play an important role in transposase binding (T. Laver and P. W. Atkinson, unpublished data). In summary, these types of transposition products containing deletions internal to the end of the element appear to be the result of non-specific cleavage events in close proximity to specific regions of transposase binding on both ends of the Hermes element.

A total of 12 deletion fragments were recovered from six progeny, with two progeny ( $\Sigma 5 \mathrm{M} \# 11$ and $\Sigma 5 \mathrm{M} \# 15)$ encompassing five of the total number of recovered transpositions of this type. Two of these progeny, $\Gamma_{\text {self }} \# 54$ and $\Delta_{\text {self }} \# 90$, also produced transposition products with breakpoints in the plasmid flanking DNA, generated clearly defined new bands in Southerns (Fig. 4a), and were previously regarded as possible germline transposition events (although it is unclear which transposition products are responsible for the integration). $\Sigma 5 \mathrm{M} \# 11$ also produces a clearly defined non-parental product in Southern blot analysis (Fig. 4b), multiple transposition products, and is also a possible germline remobilization event.

Of the remaining eight unique transpositions identified by Southern blot analysis that did not yield sequence results after TE display, three of these were assigned to be somatic events based upon the non-equimolar bands in correlation to the parental integrations. As described previously, similar banding patterns produced cut-and-paste transpositions upon TE display analysis that were believed to be the result of early somatic transposition. Among these black, with internal sequences marked in grey. The terminal nucleotides of the ITR on each end are denoted as 1 , with the internal sequences marked at ten base pair intervals from the respective end of the element. Regions of known Hermes transposase binding are denoted by the dark grey regions, and locations of degenerate (dashed arrow) or intact (arrow) GTGGC binding motifs are indicated (T. Laver and P. W. Atkinson, unpublished data)

samples were two progeny resulting from female outcrosses of the \#3M helper line. After Southern blot hybridization to both ends of the Hermes element, the $\Phi 7 \mathrm{M}$ \#47 sample was defined as a likely germline remobilization event. Three unique transpositions were detected in the 53 progeny of the $\Phi 2 \mathrm{M}$ male outcross (\#12M helper line) when probed with the Hermes right end, yet upon hybridization to the Hermes left end, no non-parental bands were detected (as shown in Fig. 4c, d). At this point it is unclear if these fragments were produced due to an incomplete, partial transposition or the result of a recombination event. A unique integration was also shared among four progeny of the $\Phi 1 \mathrm{~F}$ female outcross (\#12M helper line), but without Southern blot information for the left end of the integration or sequence obtained by TE display, one cannot definitively regard these as a germline remobilization events.

Among the Hermes transpositions recovered from the helper/reporter remobilization strategy there is evidence for local hopping of the element. Similar to the phenomena previously described for Hermes in D. melanogaster (Guimond et al. 2003) and for Mos1 in Ae. aegypti (Wilson et al. 2003), integrations were recovered in which the mobilized element inserted into itself. In the $\Delta$ self \#1 sample, two events were recovered (although one is likely somatic) that inserted into the D. melanogaster actin 5C promoter within the parental pHer [A5C-EGFP] construct. Another example, $\Delta$ self \#90, integrated within the plasmid DNA flanking the left end of the parental integration. From these events, it is evident that Hermes is capable of homing and local hopping, but the propensity of this behavior is unclear due to the lack of physical mapping of supercontigs to the Ae. aegypti chromosomes. Therefore, the question remains whether Hermes is capable of both intra- and interchromosomal transposition. As proposed by Wilson et al. (2003) in studies with the Mosl element, this would suggest that movement of the Hermes element had occurred 
during $\mathrm{S}$ phase when a second copy of the element is present. However, given the usual expression of the $A a \beta 2 \mathrm{t}$ promoter at the onset of meiosis, it is more likely that transposition occurs during segregation of the meiotic chromosomes.

The rate of likely germline remobilization using the Hermes element in Ae. aegypti occured in less than one percent of the progeny examined or approximately one in 166 progeny $(9$ in 1,491). Using a conservative approach to define a true germline event, each of the nine defined samples generated information from Southern blot analysis on one or both ends, as well as sequences demonstrating a transposition as recovered from TE display. Six of these events were recovered from self crosses with the \#3M helper line, while the remaining three transpositions were the result of male outcrosses of the \#12M helper line, and suggests that both helper lines produced sufficient levels of functional transposase. However, since other putative events were excluded on these premises and since many of the progeny analyzed did not produce a detectable band upon hybridization, this may be an underestimate for the rate of remobilization. By the inclusion of the samples that were assigned as partial integrations (based on the detection of a non-parental band on only one end of the element by Southern blot) and the integrations detected in the $\Phi 1 \mathrm{~F}$ female outcross that could not be further verified, the number of progeny containing a new integration could, at best, increase to 40 of the 1,491 examined $(2.7 \%$ or 1 in 37 progeny examined).

Non-canonical integrations into the germline of $A e$. aegypti are a feature not unique to Hermes, as other widely used transposable elements have been shown to transpose by a similar non-canonical mechanism. Although infrequent, Mos1 elements are capable of integrating into the germline of Ae. aegypti in a non-canonical fashion resulting in flanking plasmid sequence being inserted with the element (Coates et al. 1998, 2000), and increase in prevalence when using purified Mos 1 transposase in contrast to the production of transposase from a helper plasmid (Coates et al. 2000). piggyBac elements have also displayed an aberrant behavior through their insertion into large, tandem arrays in the Ae. aegypti germline (Adelman et al. 2004). Taken together, this non-canonical behavior within the mosquito germline is not specific to any given element, but in fact suggests an underlying mechanism within the Ae. aegypti germline that regulates transposition to occur in an aberrant fashion regardless of the element. Whether this is related to the recently discovered piRNA pathway that has been proposed to be a genome-wide regulator of transposition in the germline of $D$. melanogaster is unclear (Brennecke et al. 2007; Gunawardane et al. 2007). This pathway is proposed to suppress transposition primarily through slicing of the transposase transcript and so no obvious mechanistic connection with the TIRs of the transposon can be made.

The precise mechanism by which Hermes integrates into Ae. aegypti germline is still unclear, but it appears as though transposition is heavily influenced by host factors and/or the DNA repair machinery of Ae. aegypti. Aberrant integrations appear to be confined to the germline suggesting that that these host factors and/or DNA repair enzymes are not present in the soma where canonical cutand-paste transpositions are recovered. Although the exact mechanism remains cryptic, non-canonical transpositions can be attributed to an imprecise cleavage event of the element, the crucial step to initiate DNA transposition. In both types of non-canonical transposition, the cleavage step does not occur at the terminus of the element at the TIRs. Instead it is proposed that the cleavage event can occur near regions of specific or non-specific Hermes transposase binding, thus influencing the outcome of the resulting transposition product to be the result of a deletion internal to the ends of the element or a breakpoint within the flanking plasmid DNA respectively. One possible explanation for this aberrant behavior may be due to the affinity of a DNA binding protein to the TIRs of the element, thus disrupting the normal cleavage of the element by the transposase and forcing DNA cleavage to occur elsewhere.

Non-specific binding and cleavage has previously been detected with other transposable elements, such as bacteriophage $\mathrm{Mu}$, where it was found that the presence of $\mathrm{Mu}$ DNA can activate MuA transposase to transpose non-Mu DNA (Goldhaber-Gordon et al. 2002b). Using a "singleend" transposon, a second end is improvised from non- $\mathrm{Mu}$ DNA to allow for transposition to occur, and is believed to have evolved as a default pathway for when the transpososome assembles on a single end sequence (GoldhaberGordon et al. 2002b). The "pseudo-ends" generated by such transposition are highly selected for their cleavage sites (TG/CA), and resemble that of the Mu transposon and many other distantly related TEs (Goldhaber-Gordon et al. 2002a). The recognition of cryptic recombination signal sequences has also been described for Rag-1/2 recombinase in V(D)J recombination (Lewis et al. 1997), which transposes by a mechanism similar to Hermes (Zhou et al. 2004). A similar type of non-specific recognition and cleavage of non-transposon ends may also be occurring with Hermes in the mosquito germline. Aberrant transpososome assembly may result in recognition and cleavage of flanking plasmid DNA, and supports the evidence of cleavage at $\mathrm{CA}$ residues in the non-Hermes DNA as previously described.

The two types of non-canonical integrations recovered as a result of the remobilization experiments may present different outcomes for future mobility. The integrations containing flanking plasmid will more than likely be again 
able to achieve post-integration mobility since the element and its transposase binding sites are still intact as demonstrated through the parental non-canonical integration used in this study. However, it is unclear whether the element would again create a transposition product with flanking plasmid sequence or contain internal deletions to the element. In the samples recovered that contain deletions of the Hermes ends, it is unlikely that these new integrations would again be able to transpose without the necessary regions for transposase binding and likely represent a "dead end" for the element.

Acknowledgments This research was supported by PHS grant AI45741 to PWA. The authors thank Robert Hice and Stephanie Russell for technical advice and members of the Atkinson laboratory for discussions on this research. RCS thanks the Cellular, Molecular and Developmental Graduate Program for support.

Open Access This article is distributed under the terms of the Creative Commons Attribution Noncommercial License which permits any noncommercial use, distribution, and reproduction in any medium, provided the original author(s) and source are credited.

\section{References}

Adelman ZN, Jasinskiene N, Vally KJ, Peek C, Travanty EA, Olson KE, Brown SE, Stephens JL, Knudson DL, Coates CJ et al (2004) Formation and loss of large, unstable tandem arrays of the piggyBac transposable element in the yellow fever mosquito, Aedes aegypti. Transgenic Res 13:411-425

Adelman ZN, Jasinkskiene N, Onal S, Juhn J, Ashikyan A, Salampessy M, MacCauley M, James AA (2007) Nanos gene control DNA mediates developmentally regulated transposition in the yellow fever mosquito Aedes aegypti. Proc Natl Acad Sci USA 104:9970-9975

Allen ML, O'Brochta DA, Atkinson PW, Levesque CS (2001) Stable, germ-line transformation of Culex quinquefasciatus (Diptera: Culicidae). J Med Entomol 38:701-710

Anxolabehere D, Kidwell MG, Periquet G (1988) Molecular characteristics of diverse populations are consistent with the hypothesis of a recent invasion of Drosophila melanogaster by mobile P elements. Mol Biol Evol 5:252-269

Atkinson PW, Warren WD, O'Brochta DA (1993) The hobo transposable element of Drosophila can be cross-mobilized in houseflies and excises like the Ac element of maize. Proc Natl Acad Sci U S A 90:9693-9697

Brennecke JB, Aravin AA, Stark A, Dus M, Kellis M, Sachidanandam R, Hannon GL (2007) Discrete small RNA-generating loci as master regulators of transposon activity in Drosophila. Cell 128:1089-1103

Coates CJ, Turney CL, Frommer M, O'Brochta DA, Warren WD, Atkinson PW (1995) The transposable element mariner can excise in non-drosophilid insects. Mol Gen Genet 249:246-252

Coates CJ, Jasinskiene N, Miyashiro L, James AA (1998) Mariner transposition and transformation of the yellow fever mosquito, Aedes aegypti. Proc Natl Acad Sci U S A 95:3748-3751

Coates CJ, Jasinskiene N, Pott GB, James AA (1999) Promoterdirected expression of recombinant fire-fly luciferase in the salivary glands of Hermes-transformed Aedes aegypti. Gene 226:317-325
Coates CJ, Jasinskiene N, Morgan D, Tosi LR, Beverley SM, James AA (2000) Purified mariner (Mos1) transposase catalyzes the integration of marked elements into the germ-line of the yellow fever mosquito, Aedes aegypti. Insect Biochem Mol Biol 30:1003-1008

Evertts AG, Plymire C, Craig NL, Levin HL (2007) The Hermes transposon of Musca domestica is an efficient tool for the mutagenesis of Schizosaccharomyces pombe. Genetics 177:2519-2523

Goldhaber-Gordon I, Early MH, Gray MK, Baker TA (2002a) Sequence and positional requirements for DNA sites in a mu transpososome. J Biol Chem 277:7703-7712

Goldhaber-Gordon I, Williams TL, Baker TA (2002b) DNA recognition sites activate $\mathrm{MuA}$ transposase to perform transposition of non-Mu DNA. J Biol Chem 277:7694-7702

Guimond N, Bideshi DK, Pinkerton AC, Atkinson PW, O'Brochta DA (2003) Patterns of Hermes transposition in Drosophila melanogaster. Mol Genet Genomics 268:779-790

Gunawardane, L.S., Saito, K., Nishida, K., Miyoshi, K., Kawamura, Y., Nagami, T., Siomi, H., and Siomi, M.C. (2007). A slicermediated mechanism for repeat-associated siRNA $5^{\prime}$ end formation in Drosophila. Science 315

Hayes RO (1953) Determination of a physiological saline solution for Aedes aegypti. J Econ Ent 46:624-627

Horn C, Offen N, Nystedt S, Hacker U, Wimmer EA (2003) piggyBac-based insertional mutagenesis and enhancer detection as a tool for functional insect genomics. Genetics 163:647661

James AA (2000) Control of disease transmission through genetic modification of mosquitoes. In: Hander AM, James AA (eds) Insect transgenesis methods and applications. CRC, Boca Raton, FL, pp 319-333

Jasinskiene N, Coates CJ, Benedict MQ, Cornel AJ, Rafferty CS, James AA, Collins FH (1998) Stable transformation of the yellow fever mosquito, Aedes aegypti, with the Hermes element from the housefly. Proc Natl Acad Sci U S A 95:3743-3747

Jasinskiene N, Coates CJ, James AA (2000) Structure of Hermes integrations in the germline of the yellow fever mosquito, Aedes aegypti. Insect Mol Biol 9:11-18

Lawson D, Arensburger P, Atkinson P, Besansky NJ, Bruggner RV, Butler R, Campbell KS, Christophides GK, Christley S, Dialynas $E$ et al (2007) VectorBase: a home for invertebrate vectors of human pathogens. Nucleic Acids Res 35:D503-D505

Lewis SM, Agard E, Suh S, Czyzyk L (1997) Cryptic signals and the fidelity of V(D)J joining. Mol Cell Biol 17:3125-3136

Lorenzen MD, Berghammer AJ, Brown SJ, Dennell RE, Klingler M, Beeman RW (2003) piggyBac-mediated germline transformation in the beetle Tribolium castaneum. Insect Mol Biol 12:433440

Lorenzen MD, Kimzey T, Shippy TD, Brown SJ, Dennell RE, Beeman RW (2007) piggyBac-based insertional mutagenesis in Tribolium castaneum using donor/helper hybrids. Insect Mol Biol 16:265-275

Marcus JM, Ramos DM, Monteiro A (2004) Germline transformation of the butterfy Bicyclus anynana. Proc Biol Sci 271:S263-S265

Michel, K., Stamenova, A., Pinkerton, A.C., Franz, G., Robinson, A.S., Gariou-Papalexiou, A., Zacharopoulou, A., O'Brochta, D.A., and Atkinson, P.W. (2001). Hermes-mediated germ-line transformation of the Mediterranean fruit fly, Ceratitis capitata. Insect Mol Biol 10

Morris AC, Eggleston P, Crampton JM (1989) Genetic transformation of the mosquito Aedes aegypti by micro-injection of DNA. Med Vet Entomol 3:1-7

O'Brochta DA, Warren WD, Saville KJ, Atkinson PW (1996) Hermes, a functional non-drosophilid gene vector from Musca domestica. Genetics 142:907-914 
O'Brochta DA, Atkinson PW, Lehane MJ (2000) Transformation of Stomoxys calcitrans with a Hermes gene vector. Insect Mol Biol 9:531-538

O'Brochta DA, Sethuraman N, Wilson R, Hice RH, Pinkerton AC, Levesque CS, Bideshi DK, Jasinskiene N, Coates CJ, James AA et al (2003) Gene vector and transposable element behavior in mosquitoes. J Exp Biol 206:3823-3834

Pinkerton AC, Michel K, O'Brochta DA, Atkinson PW (2000) Green fluorescent protein as a genetic marker in transgenic Aedes aegypti. Insect Mol Biol 9:1-10

Robinson AS, Franz G, Atkinson PW (2004) Insect transgenesis and its potential role in agriculture and human health. Insect Biochem Mol Biol 34:113-120

Sarkar A, Coates CJ, Whyard S, Willhoeft U, Atkinson PW, O'Brochta DA (1997) The Hermes element from Musca domestica can transpose in four families of cyclorrhaphan flies. Genetica 99:15-29

Scali C, Nolan T, Sharakhov I, Sharakhova M, Crisanti A, Catteruccia F (2007) Post-integration behavior of a Minos transposon in the malaria mosquito Anopheles stephensi. Mol Genet Genomics 278:575-584

Sethuraman N, Fraser MJ Jr, Eggleston P, O'Brochta DA (2007) Postintegration stability of piggyBac in Aedes aegypti. Insect Biochem Mol Biol 37:941-951

Smith RC, Walter MF, Hice RH, O'Brochta DA, Atkinson PW (2007) Testis-specific expression of the beta2 tubulin promoter of Aedes aegypti and its application as a genetic sex-separation marker. Insect Mol Biol 16:61-71

Wilson R, Orsetti J, Klocko AD, Aluvihare C, Peckham E, Atkinson PW, Lehane MJ, O'Brochta DA (2003) Post-integration behavior of a Mos1 mariner gene vector in Aedes aegypti. Insect Biochem Mol Biol 33:853-863

Zhou L, Mitra R, Atkinson PW, Hickman AB, Dyda F, Craig NL (2004) Transposition of hAT elements links transposable elements and V(D)J recombination. Nature 432:995-1001 